\title{
MODELS OF MULTIPLE SOURCES OF ASCERTAINMENTS PER PROBAND ${ }^{1}$
}

\author{
Norikazu YASUDA \\ Division of Genetics, National Institute of Radiological Sciences, \\ Chiba 260, Japan
}

\begin{abstract}
Summary A correction parameter of sampling bias in evaluating the prevalence of rare diseases in population, the probability of ascertainment, can be estimated by a surveillance system where multiple sources shall ascertain independently affected persons as proband in several times. A crucial observation in estimation of the ascertainment probability is the mean number of ascertainment per proband. Two models were presented for constant and variable probability of ascertainment with Poisson law and the logarithmic series distributions, respectively. The variable model yields somewhat smaller estimate of the ascertainment probability than the one obtained from the constant model. However the magnitudes of difference between two estimates seem not so appreciable that the ascertainment probability obtained from the logseries model may be good for evaluating the prevalence of rare diseases. A numerical example is given.
\end{abstract}

\section{INTRODUCTION}

Human geneticists are well aware of bias in estimation of segregation frequency introduced by a way of family studies when their interest was focused on the mode of Mendelian inheritance for a rare trait in question. The correction of bias is used to be made the ascertainment probability, designated by $\pi$. When there was only one proband in each family, it is single ascertainment or $\pi=0$ since all proband were removed from the analysis. On the other hand, $\pi=1$ or complete ascertainment if all affected individuals were proband. Between two extremes it is called multiple ascertainment or $0<\pi<1$. Currently, the probability of ascertainment is used to be estimated from the distribution of probands among affected sibs, particularly multiplex families, since simplex cases are necessarily probands (Fisher, 1934). If most cases are sporadic because of a small size of siblings, a trend being observed in industrialized countries or of the trait in question being so rare, even

Received April 28, 1981

1 This study was supported in part by the grants for Project on the monitoring of congenital anomaties and for the Intractable Diseases from the Ministry of Health and Welfare of Japan. 
a large sample will give little information about the ascertainment probability.

The probability of ascertainment is in fact the probability that affected individuals in population were detected to be proband by medical examinations, it becomes of very important if the diseases were rare as usual cases in genetic disorders as well as congenital anomalies. Multiple sources of ascertainment per proband are an alternative way for obtaining information about $\pi$ (Morton, 1959, 1962). It has been said that ascertainment of proband by one source tends to make another less likely, as one physicians' referral tends to preclude anothers. On the other hand, the distribution of probands on the assumption of constant ascertainment of probands may overestimate $\pi$ because two or more siblings may be examined, and counted as probands, even though only one of them would have been submitted to examination independently.

In this paper, two models based on materials without identifying the sources shall be presented. Alternative model where the sources of ascertainment were known was found in Morton (1962) and Yasuda (1979).

\section{THE MODELS}

The constant probability model:-The 0-truncated Poisson distribution (Morton, 1959). Suppose that a proband can be ascertained in an indefinitely large number of ways. If $p_{i}$ is the probability of ascertainment from the $i^{\text {th }}$ source and there are $\mathrm{T}$ independent sources, with $\phi=\sum_{\mathrm{i}=1}^{\mathrm{T}} \mathrm{p}_{\mathrm{i}}$, the probability of ascertainment by $\mathrm{t}$ times is shown to be approximated by the Poisson law

$$
P(t)=\frac{\phi^{t} e^{-\phi}}{t !} \quad \text { as } T \rightarrow \infty
$$

The ascertainment probability under these conditions is

$$
\pi=1-\mathrm{p}(0)
$$

so that $\phi=-\ln (1-\pi)$. The 0 -truncated Poisson probability may therefore be written

$$
P(t)=\frac{[-\ln (1-\pi)]^{t}}{t !} \cdot \frac{(1-\pi)}{\pi}
$$

with the scores of maximum likelihood

and

$$
\mathrm{U}=\frac{\mathrm{A}}{\pi(1-\pi)}-\left[\frac{\overline{\mathrm{t}} \pi}{-\ln (1-\pi)}-1\right]
$$

$$
K=\frac{A[-\pi / \ln (1-\pi)-(1-\pi)]}{[\pi(1-\pi)]^{2}}
$$


in which $A$ is the total number of probands and is $\sum \mathrm{a}_{\mathrm{t}}$, a sum of the observed numer of probands who were ascertained independently $\mathrm{t}$ times, and $\overline{\mathrm{t}}=\sum \mathrm{ta}_{\mathrm{t}} / \mathrm{A}$ or mean number of ascertainment per proband (Correcting misprints in (10) and (11) of Yasuda (1979)). Maximum likelihood solution $\hat{\pi}$ satisfies the equation $\mathrm{U}=0$ or

$$
\overline{\mathrm{t}}=-\ln (1-\hat{\pi}) / \hat{\pi}
$$

A numerical table for solving the above equation has been given in Yasuda (1979).

The variable probability models. Suppose that the expected mean number of ascertainment $\phi$ is different for each proband, and has a gamma probability density

$$
\mathrm{f}(\phi)=\phi^{\mathrm{k}-1} \exp [-\dot{\phi} / \mathrm{p}] /\left[\mathrm{p}^{\mathrm{k}}(\mathrm{k}-1) !\right] \quad(0<\phi<\infty) .
$$

Where $\mathrm{p}=\mathrm{V} / \bar{\phi}$ and $\mathrm{k}=\bar{\phi}^{2} / \mathrm{V}$, and $\bar{\phi}$ and $\mathrm{V}$ are respectively an average and the variance of $\phi$. This mathematically represents heterogeneity of $\pi$ among probands, and still assumed the ascertainment be independent. It also assumes that the expected mean number of ascertainments varies continuously around a single mode*. Sometimes affection is mixture of mild and severe forms. If they are sharply discontinuous, the expected number of ascertainments may be bimodal. In such cases, reliable genetic analysis requires separation of the different entities.

The distribution of the number of ascertainments then becomes

$$
\mathrm{P}(\mathrm{t})=\int_{0}^{\infty} \mathrm{f}(\phi) \phi^{\mathrm{t}} \mathrm{e}^{-\phi} / \mathrm{t} ! \mathrm{d} \phi=\frac{(\mathrm{k}+\mathrm{t}-1) !}{(\mathrm{k}-1) ! \mathrm{t} !}\left[\frac{1}{1+\mathrm{p}}\right]^{\mathrm{k}}\left[\frac{\mathrm{p}}{1+\mathrm{p}}\right]^{\mathrm{t}}
$$

which is a negative binomial distribution (Fisher et al., 1943). Since the distribution must be truncated at zero and noting that $\pi=1-\mathrm{P}(0)=1-[1 /(1+\mathrm{p})]^{\mathrm{k}}$, we arrived at a 0 -truncated negative binomial distribution:

$$
\mathrm{P}(\mathrm{t})=\frac{(\mathrm{k}+\mathrm{t}-1) !}{(\mathrm{k}-1) ! \mathrm{t} !}\left[1-(1-\pi)^{1 / \mathrm{k}}\right]^{\mathrm{t}} \mathrm{t} \frac{1-\pi}{\pi}
$$

In his segregation analysis Morton (1969) has first used this model with somewhat different expression. A method of maximum likelihood estimation of $\mathrm{k}$ and $\mathrm{w}=$ $1 /(1+p)$ may be found in Sampford (1955). Then an approximate variance of $\pi$ will be

$$
\mathrm{V}(\hat{\pi})=(1-\hat{\pi})^{2}\left[(\ln \mathrm{w})^{2} \mathrm{~V}(\mathrm{k})+\left(\frac{\mathrm{k}}{\mathrm{w}}\right)^{2} \mathrm{~V}(\mathrm{w})+\frac{2}{\mathrm{w}} \operatorname{Cov}(\mathrm{w}, \mathrm{k})\right]
$$

in which $V($.$) and \operatorname{Cov}(.,$.$) are elements of the variance-covariance matrix.$

* If $\mathrm{k}>1$, a mode exists at $\phi=(\mathrm{k}-1) \mathrm{p}$. This infers that multiple ascertainment of probands is common. On the other hand, single ascertainment is not unusal if $k \leqq 1$ because of mode being at $\phi=0$. 
A special case for $k=1$ is of interest for its mathematical simplicity. The distribution is known as being 0-truncated geometric;

$$
\mathrm{P}(\mathrm{t})=(1-\pi) \pi^{\mathrm{t}-1} \quad(\mathrm{t}=1,2, \cdots)
$$

with the scores of maximum likelihood

$$
\mathrm{U}=\mathrm{A}[(\overline{\mathrm{t}}-1) / \pi-1 /(1-\pi)]
$$

and

$$
\mathrm{K}=\mathrm{A} /\left[\pi(1-\pi)^{2}\right] \text {. }
$$

The maximum likelihood solution is now

$$
\hat{\pi}=1-1 / \vec{t}
$$

In practice however the nuisance parameter $\mathrm{k}$ cannot be assumed but should be estimated from data. Even so, experiences indicate that $\mathrm{k}$ is awkward in estimation processes, very often failed in convergence of iterative processes. Therefore we look for alternative models which is free of $\mathrm{k}$ and might be compatible with real circumstances.

The variance of negative binomial distribution is able to express in terms of the mean $(m)$ and $k$; namely, $V=m+m^{2} / k$. Clearly the smaller the value of $k$ the greater the variance: but, if $\mathrm{k}$ tends to be large or $\mathrm{V} \rightarrow \mathrm{m}$, and we see that, as for the Poisson distribution, the mean and variance are equal, the case of constant probability of ascertainment. If heterogeneity of the expected number of ascertainment among probands is very great, $\mathrm{k}$ becomes small and approaches its limiting value zero. This implies as $\mathrm{k} \rightarrow 0, \pi=1-[1 /(1+\mathrm{p})]^{\mathrm{k}} \rightarrow 0$ for fixed $\mathrm{p}$; namely, it approaches to the single ascertainment. However what is in fact assumed is not that $\mathrm{k}=0$ but merely that $\mathrm{k}$ was very small indeed. The parameter $\mathrm{k}$ is actually an intrinsic property of the population sampled. The situation in which a large number of anomalies are so rare that they have a small chance of being included in the sample may be well represented by the limiting form of the negative binomial distribution when $\mathrm{k}$ tends to zero.

Let $\mathrm{k}$ tend to zero, that is,

$$
\begin{aligned}
P(t) & =\lim _{\mathrm{l} \rightarrow 0} \frac{k(1-\pi)}{\pi} \cdot \frac{(k+1)(k+2) \cdots(k+t-1)}{t !}\left[\frac{p}{1+p}\right]^{t} \\
& =\frac{1}{\ln (1+p)} \cdot \frac{1}{t}\left[\frac{p}{1+p}\right]^{t} \quad(t=1,2, \cdots)
\end{aligned}
$$

since

$$
\lim _{k \rightarrow 0} \frac{k(1-\pi)}{\pi}=\lim _{k \rightarrow 0} \frac{k}{(1+p)^{k}-1}=\frac{1}{\ln (1+p)}
$$



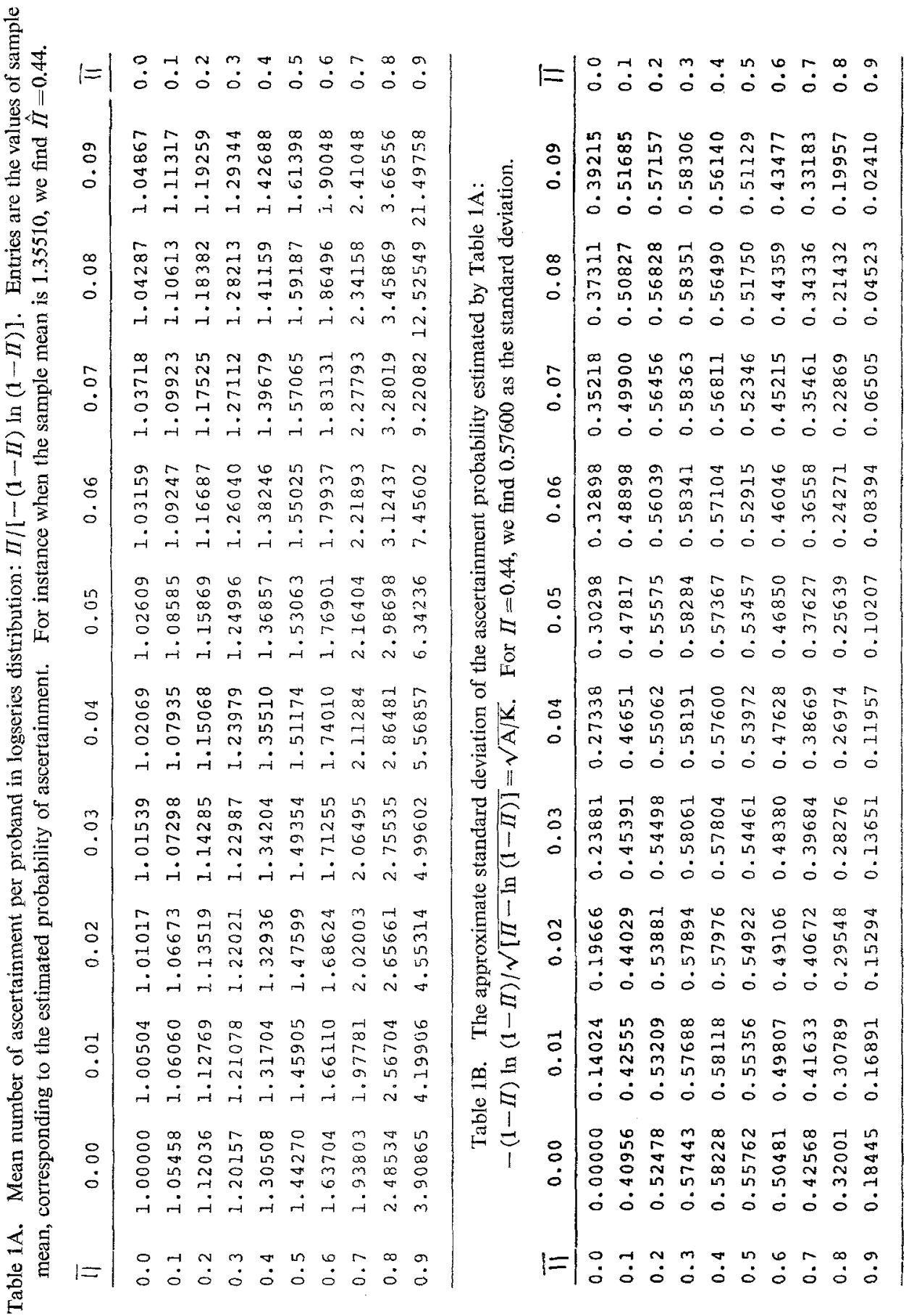

Vol. 27, No. 1, 1982 
in virture of the rule known as l'Hopital's. This is a distribution of the logarithmic series (or logseries for brevity), first derived by Fisher et al. (1943) in study of the abundance of different species.

Putting $\mathrm{p} /(1+\mathrm{p})=\Pi$, we have

$$
\mathrm{P}(\mathrm{t})=\frac{1}{-\ln (1-\Pi)} \frac{I I \mathrm{t}}{\mathrm{t}} \quad(\mathrm{t}=1,2, \ldots)
$$

with the scores of maximum likelihood

$$
\mathrm{U}=\frac{\mathrm{A} \overline{\mathrm{t}}}{\Pi}+\frac{\mathrm{A}}{(1-\Pi) \ln (1-\bar{I})}
$$

and

$$
\mathrm{K}=\mathrm{A}[-\ln (1-\Pi)+\Pi] /[(1-\Pi) \ln (1-\Pi)]^{2} .
$$

The solution of maximum likelihood equation satisfies

$$
\overline{\mathrm{t}}=-\hat{\Pi} /[(1-\hat{I}) \ln (1-\hat{I})]
$$

In Tables $1 \mathrm{~A}$ and $1 \mathrm{~B}$ tabulated respectively the mean $(\overline{\mathrm{t}})$ and the standard deviation $(\sqrt{\mathrm{A} / \mathrm{K}})$ for $\Pi=0.00$ through 0.99 with the increment 0.01 . Patil (1962) has suggested a conventional formula

$$
\Pi^{*}=1-\mathrm{p}_{1} / \overrightarrow{\mathrm{t}} \text { where } \mathrm{p}_{1}=\mathrm{a}_{1} / \mathrm{A}
$$

The parameter $I I$ is in fact correspond to $\pi$ in the geometric model, but $I$ is freed from the nuisance parameter $\mathrm{k}$. Also, it is the probability of 'presence' of affected persons. It has been shown that the magnitude of $p /(1+p)$ in the 0 -truncated negative binomial distribution depends only on the size of the sample taken from the population (Pielou, 1977). Thus if we increase the area from which probands are taken or prolong the duration of sampling, the only effect will be to change the value of $I=\mathrm{p} /(1+\mathrm{p})$, provided that the sample still comes from the same population. The parameter $I I$ therefore can be inferred to a mean probability of ascertainment taking into account heterogeneity to be proband.

The constant probability model involving probands from multiple sources only. If most cases are sporadic, even a large sample will give little information about the ascertainment probability. The geneticist may concentrate on multiple sources of ascertainment or there were two or more sources per proband. Under this circumstance, the Poisson distribution is truncated at $\mathrm{t}=0$ and 1 ; namely,

$$
P(t)=\frac{[-\ln (1-\pi)]^{t}(1-\pi)}{t ![\pi+(1-\pi) \ln (1-\pi)]} \quad(t=2,3, \cdots)
$$



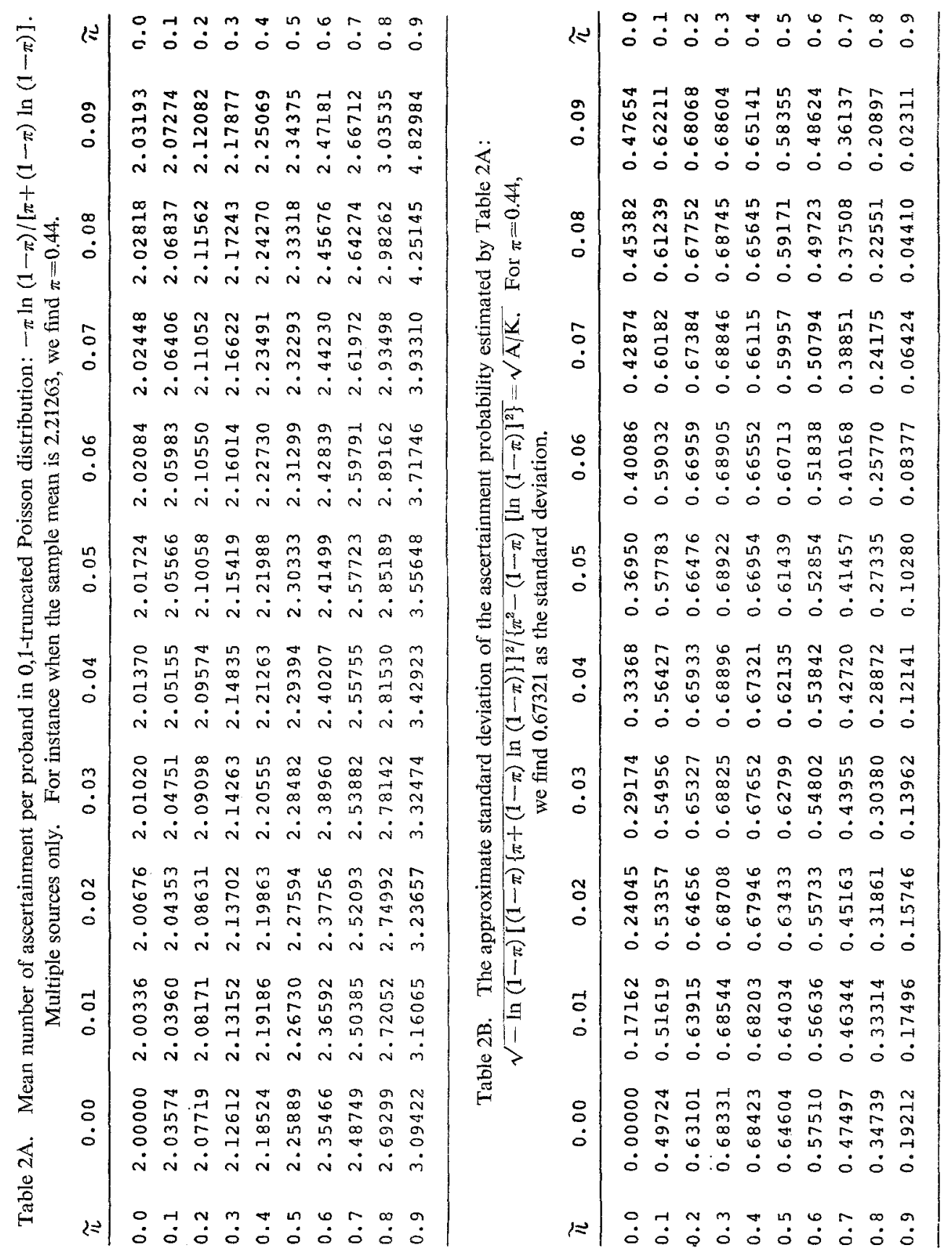

Vol. 27, No. 1, 1982 
The scores are then

$$
U=\frac{-\mathrm{A}}{1-\pi}\left[\frac{\overline{\mathrm{t}}}{\ln (1-\pi)}+\frac{\pi}{\pi+(1-\pi) \ln (1-\pi)}\right]
$$

and

$$
\mathrm{K}=\mathrm{A} \cdot \frac{\pi^{2}-(1-\pi)[\ln (1-\pi)]^{2}}{-\ln (1-\pi)[(1-\pi)[\pi+(1-\pi) \ln (1-\pi)\}]^{2}}
$$

The maximum likelihood solution satisfies

$$
\overline{\mathrm{t}}=-\hat{\pi} \ln (1-\hat{\pi}) /[\hat{\pi}+(1-\hat{\pi}) \ln (1-\hat{\pi})]
$$

which has been tabulated in Table $2 \mathrm{~A}$ for the value $\overrightarrow{\mathrm{t}}$ for $\pi=0.00$ through 0.99 with the increment 0.01 . The standard deviation of $\hat{\pi}$ is also tabulated in Table 2B.

The variable probability model involving probands from multiple sources. Occasionally, probands ascertained once were not reported because of interest of investigators directed to multiple sources of ascertainment. An alternative model is the 1 -truncated logseries distribution; that is

$$
\mathrm{P}(\mathrm{t})=\Pi \mathrm{t} /[\mathrm{S} \cdot \mathrm{t}] \quad(\mathrm{t}=2,3, \cdots)
$$

in which $\mathrm{S}=-\ln (1-\Pi)-\Pi$. The scores of maximum likelihood are

$$
\mathrm{U}=\mathrm{A}[\overline{\mathrm{t}} / \Pi-\Pi /\{\mathrm{S}(1-\Pi)\}]
$$

and

$$
\mathrm{K}=\mathrm{A}\left[\mathrm{S}(2-\Pi)-\Pi^{2}\right] /[\mathrm{S}(1-\Pi)]^{2} .
$$

The solution of the equation, $U=0$, satisfies

$$
\overline{\mathrm{t}}=\hat{\Pi}^{2} /[\hat{\mathrm{S}}(1-\hat{\Pi})] \text {. }
$$

Tables $3 \mathrm{~A}$ and $3 \mathrm{~B}$ are respectively tabulated for the value of $\overline{\mathrm{t}}$ and the standard deviation of $\hat{I}$ for $I=0.00$ through 0.99 with the increment 0.01 . An approximate estimating formula similar to the one suggested by Patil (1962) may be obtained from

$$
I *=1-2 \mathrm{p}_{2} / \overline{\mathrm{t}} \text { with } \mathrm{p}_{2}=\mathrm{a}_{2} / \mathrm{A},
$$

where $a_{2}$ is the number of probands ascertained twice.

\section{NUMMERICAL EXAMPLE}

Albinism. This is an example of nearly complete ascertainment. Tanaka and Watanabe (1967) have reported on the number of ascertainments without identifying the sources for albino probands in the eastern part of Shizuoka prefecture, and 

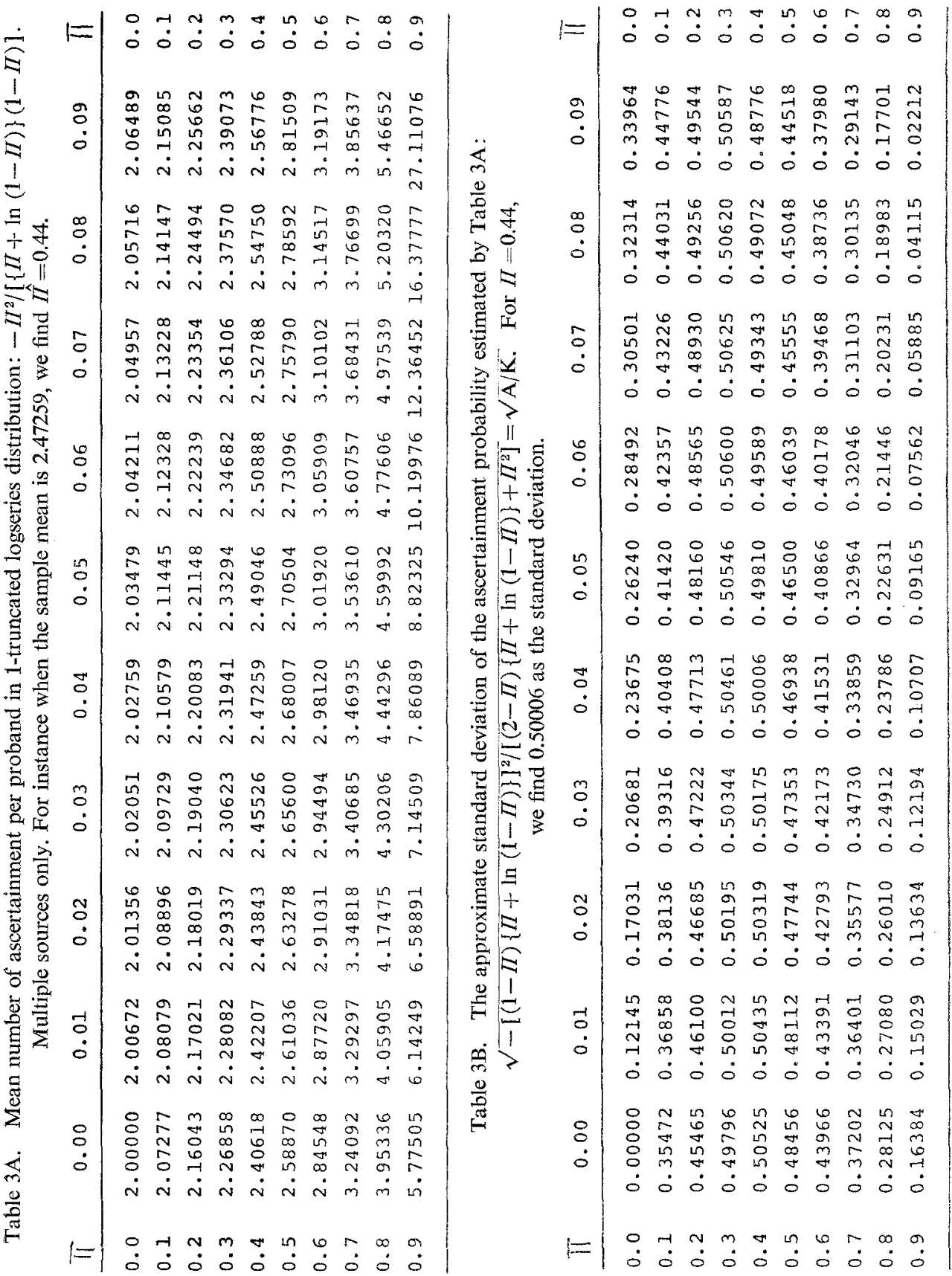

Vol. 27, No. 1,1982 
found $a_{1}=2, a_{2}=1$ and $a_{3}=5$ so that $A=8$. Although an apparent bimodality of the distribution suggests that once albino person ascertained as proband he is likely to be reported repeatedly as proband, this may serve for an illustration of the models. The observed mean number of ascertainments per proband is $\vec{t}=19 / 8=$ 2.375 which leads to $\hat{\pi}=0.87 \pm 0.07$ (see Table 1A of Yasuda (1979)) in the constant model and $\hat{I}=0.78 \pm 0.12$ in the variable model. In fact 0.78 is found in Table $1 \mathrm{~A}$ and $0.12=0.343 / \sqrt{8}$ in Table $1 \mathrm{~B}$. The variable model yields somewhat lower estimate. $\hat{\pi}=(1-1 / 2.375)=0.58 \pm 0.11$ may be obtained from a geometric probability model, a special case of the negative binomial law $(\mathrm{k}=1)$. This value is the smallest, but the standard error is intermediate. Table 4 is a summary of tests for goodness of fit to three models. This particular example is fitted to neither model because of an excess frequency of the class with the number of ascertainment being three. If we assume the excess is in fact due to lump three or more times of ascertainment, the data fit to all model.

If the first class of observation is removed from the analysis, the ascertainment probability becomes to $0.84 \pm 0.11$ for constant model (use Tables $2 \mathrm{~A}$ and $2 \mathrm{~B}$ ), $0.60 \pm 0.18$ for the variable model (use Tables $3 \mathrm{~A}$ and $3 \mathrm{~B}$ ), and $0.65 \pm 0.12$ for the geometric probability model. But, no degree of feedom is left for tests of goodness for fit. The rejection of probands ascertained once from the analysis resulted underestimation of the ascertainment probability.

\section{DISCUSSION}

In Fig. 1 the theoretical probabilities of ascertainment were compared for three different models for a given mean number of ascertainment per proband. Poisson law always gives the highest estimate, followed by the logseries and the geometric model comes the last. These were in fact observed in the above example. Some cases of the negative binomial distribution are also included in the Fig. 1 for the sake of comparison. It can be seen that when the nuisance parameter $\mathrm{k}$ tends to $\infty$ the curve approaches to Poisson case while $\mathrm{k} \rightarrow 0$ the ascertainment probability tends to be zero, whatsoever the mean number of ascertainment per proband is, as far as the negative binomial law retains. Although the negative binomial distribution is so mathematically general that the model may be of theoretically interest, the model for $\mathrm{k} \rightarrow 0$ seems not to be appropriate for describing the method of ascertainment.

In sampling surveys if the probabilities of selection of affected persons as proband is proportional to the number of ascertainment, for instance, the severe the disorder, the more often to be repeatedly proband, then the distribution to which the sample corresponds is

$$
P^{\prime}(t)=\frac{t P(t)}{\sum t P(t)}
$$




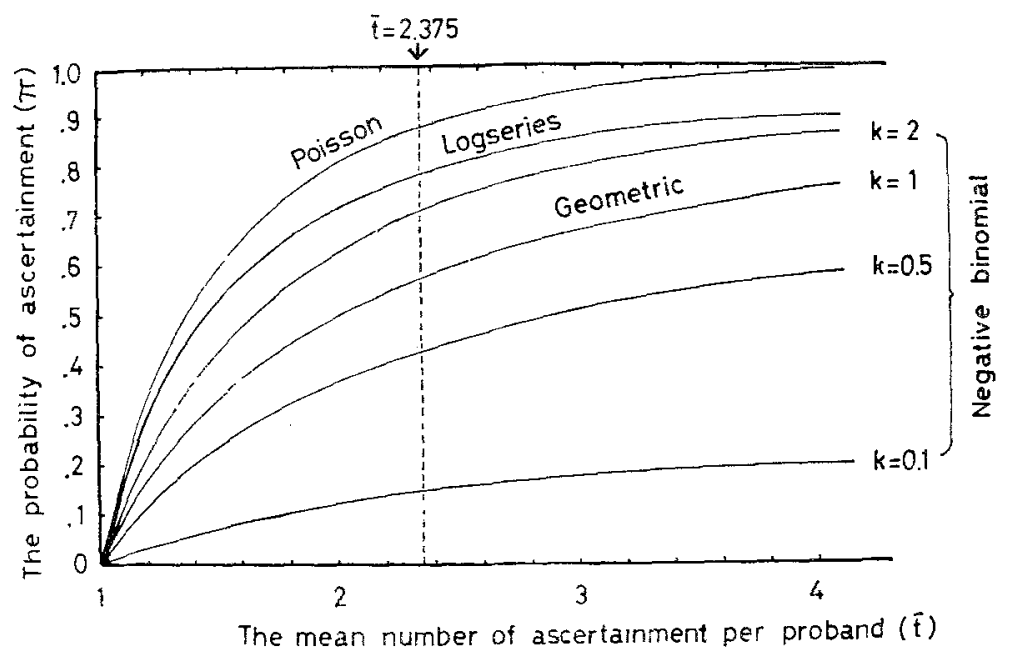

Fig. 1. Relation between the mean number of ascertainment per proband and the probability of ascertainment under the models of Poisson, logseries and negative binomial distribution.

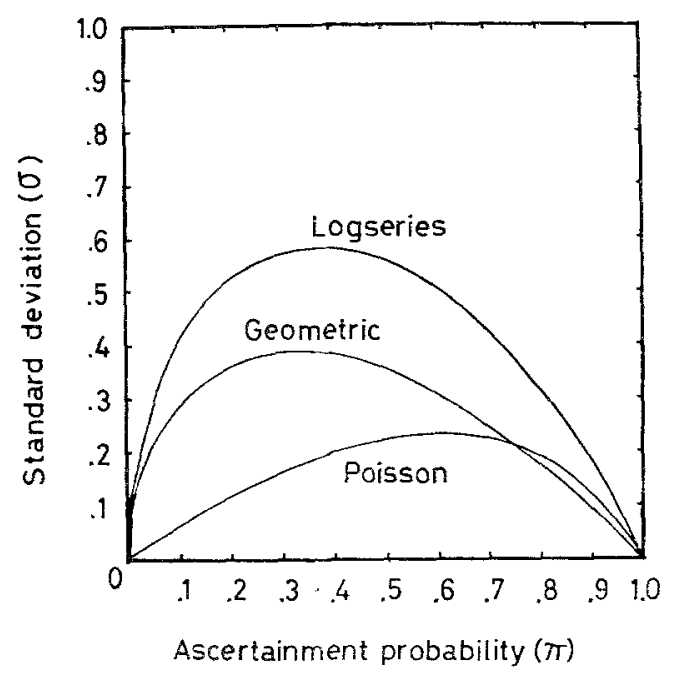

Fig. 2. Standard deviation of the ascertainment probability under the models of Poisson, geometric and logseries distribution.

It is easily verified that $\mathrm{P}^{\prime}(t)$ is geometric when $\mathrm{P}(\mathrm{t})$ is logseries (Rao, 1963). Thus, even so the observed distribution sometimes does fit to a geometric, which is a special case of the negative binomial distribution, the actual distribution may well be logseries.

Figure 2 depicts the standard deviation of estimated probability of ascertain- 
Table 4. Ascertainment distribution of albinism and test for goodness of fit to three models.

\begin{tabular}{cccccc}
\hline $\mathrm{t}$ & obs & $\begin{array}{c}\text { Poisson } \\
\exp \left(\chi^{2}\right)\end{array}$ & $\begin{array}{c}\text { Geometric } \\
\exp \left(\chi^{2}\right)\end{array}$ & $\begin{array}{c}\text { Logseries } \\
\exp \left(\chi^{2}\right)\end{array}$ \\
\hline 1 & 2 & $2.38(0.06)$ & $3.36(0.55)$ & $4.07(1.05)$ \\
2 & 1 & $2.47(0.88)$ & $1.13(0.01)$ & $1.57(0.21)$ \\
3 & 5 & $1.71(6.32)$ & $0.66(28.54)$ & $0.80(22.05)$ \\
$\geqq 4$ & 0 & $1.44(1.44)$ & $2.88(2.88)$ & $1.56(1.56)$ \\
\hline Total & 8 & $(8.69)^{\mathrm{a}}$ & $(31.98)^{\mathrm{a}}$ & $(24.87)^{\mathrm{a}}$ \\
& & $(2.02)^{\mathrm{b}}$ & $(1.16)^{\mathrm{b}}$ & $(3.59)^{\mathrm{b}}$ \\
\hline
\end{tabular}

$t$, number of sources. a Value of chi-square with 2 degrees of freedom. b Value of chi-square with one degree of freedom when the last two classes are lumped.

ment. The largest standard deviation of the logseries distribution is a reflection of heterogeneity in $\Pi$. Figures 1 and 2 may also be used for obtaining graphically the estimate and the standard deviation of the ascertainment probability from an observed mean number of the ascertainment, respectively. Thus, for $\bar{t}=2.375$ we have the estimate 0.87 for the Poisson law, 0.78 for the logseries and 0.58 for the geometric. The standard deviation may read from the Fig. 2 for the estimates obtained from Fig. 1. Dividing them by $\sqrt{8}$ or the square root of the total number of probands, we obtain the standard error as given in Table 4.

In practice the observed mean number of ascertainment seldom exceeds 2 , and which is nearly complete ascertainment. This implies the ascertainment probability being less than 0.8 in the constant model and 0.72 in the variable model (Fig. 1). Thus the differences in the ascertainment probability between two models are at best order of 0.1, and such magnitude is in fact within the standard deviation (Fig. 2). Although such difference in the estimates of the ascertainment probability is tolerable for testing as well as estimating the segregation frequency, the prevalence will vary within the range of ten percent. That is, the prevalence may be obtained from

$$
\left.\mathrm{P}=\mathrm{A} / \pi \pm \mathrm{A} \cdot \mathrm{S}_{\pi} / \pi^{2} \quad \text { (Barrai } \text { et al., } 1965\right)
$$

For albinism we have $10 \pm 1$ for the constant model, $11 \pm 2$ for the variable model, and $14 \pm 3$ for the geometric probability model. Three estimates of prevalence are in all within the standard error.

In the above considerations, we do assume that the probands were ascertained independently $\mathrm{t}$ times. Intuitively, violation of this assumption leads to a higher estimate of the ascertainment probability, but the order of magnitude is unknown.

In summary the logarithmic series distribution may be good for describing the heterogeneity of the ascertainment probability. The Poisson distribution yields an upper estimate whereas the logseries probability model may give a lower value. However, the differences in magnitude are not so appreciable. 
Acknowledgements I wish to thank Mr. K. Fukuhisa for his technical assistance in running computer programs on ACOS-700S installed in the National Institute of Radiological Sciences.

\section{REFERENCES}

Barrai, I., Mi, M.P., Morton, N.E., and Yasuda, N. 1965. Estimation of prevalence under incomplete selection. Am. J. Hum. Genet. 17: 221-236.

Fisher, R.A. 1934. The effect of method of ascertainment upon the estimation of frequencies. Ann Eugen. 6: 13-25.

Fisher, R.A., Corbet, A.S., and Williams, C.B. 1943. The relation between numbers of species and the number of individuals in a random samples of an animal population. J. Anim. Ecol. 12: $42-58$.

Morton, N.E. 1959. Genetic tests under incomplete ascertainment. Am. J. Hum. Genet. 11: 1-16.

Morton, N.E. 1962. Segregation and linkage. In Methodology in Human Genetics (W.J. Burdette, ed.). Holden-Day, Inc., San Francisco. pp. 17-52.

Morton, N.E. 1969. Segregation analysis. In Computer Applications in Genetics (N.E. Morton, ed.). University Press of Hawaii, Honolulu. pp. 129-139.

Patil, G.P. 1962. Some methods of estimation for the logarithmic series distribution. Biometrics 18: $68-75$.

Pielou, E.C. 1977. Mathematical Ecology. John Wiley and Sons, New York, pp. 269-290.

Rao, C.R. 1963. On discrete distributions arising out of method of ascertainment. In Proc. Int. Symp. on Classical and Contagious Discrete Distributions. G.P. Patil, ed., Statistical Publishing Society, Calcutta. pp. 320-332.

Sampford, M.R. 1955. The truncated negative binomial distribution. Biometrika 42: 58-69.

Tanaka, K., and Watanabe, K. 1967. Frequency of albino in Japanese population. Jpn. J. Human Genet. 11: 231-243.

Yasuda, N. 1979. Estimation of the ascertainment probability of rare disease. Jpn. J. Human Genet. 24: 265-291. 